\title{
EFFECT OF SHILAJIT ON EXPERIMENTAL MODELS OF INFLAMMATORY BOWEL DISEASE IN RATS
}

\author{
NEELIMA S. , NARESH BABU T., PRADEEP KUMAR M. \\ Department of Pharmacology, Vasavi Institute of Pharmaceutical Sciences, Kadapa \\ Email: neelus1989@gmail.com
}

Received: 27 Jan 2017, Revised and Accepted: 20 Apr 2017

\begin{abstract}
Objective: Inflammatory bowel disease (IBD) is a chronic condition of the intestine with unknown etiology involving multiple immunes, genetic and environmental factors. Oxidative stress is believed to be a key factor in the pathogenesis and perpetuation of the mucosal damage in IBD. The present study is to elucidate the effects of shilajit extract on the extent and severity of enterocolitis induced by subcutaneous administration of Indomethacin $(7.5 \mathrm{mg} / \mathrm{kg} \mathrm{b.} \mathrm{w)} \mathrm{in} \mathrm{Wistar} \mathrm{rats.}$
\end{abstract}

Methods: Study comprised of 6 groups ( $\mathrm{n}=6)$, normal vehicle control, indomethacin-induced $(7.5 \mathrm{mg} / \mathrm{kg}$, 2days), shilajit alone $50 \mathrm{mg} / \mathrm{kg} \mathrm{b}$. w, shilajit treated groups ( 25 and $50 \mathrm{mg} / \mathrm{kg}, \mathrm{p}$. o) and sulfasalazine treated $\left(100 \mathrm{mg} / \mathrm{kg}\right.$, p. o) groups. Drug treatment continued for $11 \mathrm{~d}$ and on $12^{\text {th }} \mathrm{d}$ scarification was done. The colonic mucosal injury was assessed by macroscopic scoring, biochemical (LDH, MPO, GSH and LPO) tests were performed.

Results: Pretreatment with shilajit showed a decrease in macroscopic scores, LDH, MPO, LPO and elevation levels of GSH as compared to the indomethacin-treated group.

Conclusion: The present study suggests that the protective effect of shilajit in indomethacin-induced enterocolitis might be attributed to its scavenging effect on oxygen-derived free radicals and may be beneficial in patients with inflammatory bowel disease.

Keywords: Inflammatory bowel disease, Shilajit, Indomethacin, Subcutaneous

(c) 2017 The Authors. Published by Innovare Academic Sciences Pvt Ltd. This is an open access article under the CC BY license (http://creativecommons.org/licenses/by/4.0/) DOI: http://dx.doi.org/10.22159/ijcpr.2017v9i4.20756

\section{INTRODUCTION}

Inflammatory bowel disease (IBD) is a chronic recurrent disease characterized by intestinal mucosal inflammation and includes ulcerative colitis (UC) and Crohn's disease (CD). The pathogenesis of IBD is a multifactorial process [1]. UC and CD are the two major phenotypes. These diseases have a great impact on the quality of life of the affected persons and their families. UC is characterized by confluent mucosal inflammation of the colon starting at the anal verge and extending proximally for a variable extent $(e . g$., proctitis, left-sided colitis, or pancolitis). CD, by contrast, is characterized by transmural inflammation of any part of the gastrointestinal tract but, most commonly in the area adjacent to the ileocecal valve. Medical therapy for IBD is challenging because no unique abnormality has been identified. For many years Glucocorticoids and Sulfasalazine (SLZ) were the mainstays of medical therapy for IBD2. Infliximab, a monoclonal antibody against TNF- $\alpha$ has been proved highly effective in the clinical management of both forms of IBD. Considering oxidative stress is one of the factors in IBD, antioxidants could be likely to provide relief [2].

Shilajit is a herbo-mineral drug, blackish-brown exudation, obtained as a mineral resin or as a plant fossil composed of humus and organic plant material that has been compressed by layers of rock mixed with microbial metabolites [3]. It is found in the serene surroundings of Himalayas. The common names are vegetable asphalt, mineral pitch, mountain sweat, mountain oil, rock juice. Shilajit is an important drug of the ancient Hindu materia medica and is extensively used by the Hindu physicians to treat a variety of diseases. It is said to be efficacious against phthisis, chronic bronchitis, asthma, digestive troubles, sexual and bladder calculi, dropsy, nervous diseases, leprosy, diabetes, and fracture of bones. It is also used in parasitic diseases of the skin and as an antiphlogistic. The biologically important classes of compounds of shilajit include dibenzo-alpha pyrones, phospholipids, triterpenes and phenolic acids of low molecular weight, humins and humic acids, fulvic acids which are known as "carrier molecules" and trace elements (Fe, Ca, Cu, Zn, Mg, Mn, Mo, P) [4].

Shilajit has been reported for its anti-ulcerogenic and antiinflammatory, antioxidant, antidiabetic, memory enhancement, anxiolytic, antistress, immunomodulatory and anti-allergic activity [5]. Although the majority of natural drugs are derived from plant and animal origins, a few of them, obtained from mineral sources, like shilajit, are of paramount significance as pharmaceutical aids. No scientific data regarding the activity of shilajit on IBD is available. Hence, the present study was designed to evaluate the effect of shilajit on experimental models of IBD in rats. The objective of the study is to investigate the effect of shilajit for treating IBD in rats by using indomethacin-induced enterocolitis by estimating macroscopic sores and enzyme markers such as lactate dehydrogenase (LDH), myeloperoxidase (MPO), lipid peroxidase (LPO), glutathione (GSH) and compare effect of shilajit with that of standard drug i.e. Sulfasalazine (SLZ) in the above model.

\section{MATERIALS AND METHODS}

Albino Wistar Rats of either sex weighing 150-200 g were used for the present study. The animals were collected from animal house. The animals were maintained under controlled conditions of temperature $\left(22 \pm 2{ }^{\circ} \mathrm{C}\right)$, humidity $(50 \pm 5 \%)$ and $12 \mathrm{~h}$ light-dark cycles. All the animals were acclimatized for seven ds before the study. The animals were randomized into experimental and control groups and housed individually in sanitized polypropylene cages containing sterile paddy husk as bedding. They had free access to standard pellets as basal diet and water ad libitum. Animals were habituated to laboratory conditions for $48 \mathrm{~h}$ prior to the experimental protocol to minimise if any of non-specific stress. All the studies conducted were approved by the Institutional Animal Ethical Committee (IAEC) of Sree Siddaganga College of Pharmacy, Tumkur, Karnataka. Approval No. SSCPT, IAEC, Clear 110/2011-12 dated 30/11/2011, according to prescribed guidelines of Committee for the Purpose of Control and Supervision of Experiments on Animals (CPCSEA), Government of India.

\section{Preparation of suspension}

Shilajit was dissolved in distilled water and SLZ was suspended in distilled water using sodium carboxymethyl cellulose (sodium CMC, $0.3 \%$ ). Both these suspensions were administrated orally to the animals with the help of an intragastric catheter. 


\section{Experimental methods}

The male Wistar rats (200-250 gm) were selected and randomized in to following groups [6]. Small intestinal inflammation was induced by two subcutaneous injections of Indo $(7.5 \mathrm{mg} / \mathrm{kg}$, s. c) spaced $24 \mathrm{~h}$ apart. The study comprised of six different groups each consisting of six animals as follows:

Group I: Normal control animals. (Vehicle control)

Group II: Animals received only Indo $(7.5 \mathrm{mg} / \mathrm{kg}$, s. c.) on two consecutive ds. (Positive control)

Group III: Received pretreatment with shilajit (50 mg/kg bw. p. o) till 11th d.

Group IV: Received pretreatment with shilajit ( $25 \mathrm{mg} / \mathrm{kg}$ bw. p. o), followed by Induction with Indo $(7.5 \mathrm{mg} / \mathrm{kg}, \mathrm{s} . \mathrm{c})$ on 8 th and 9 th $\mathrm{d}$ of treatment.
Group IV: Received pretreatment with shilajit ( $50 \mathrm{mg} / \mathrm{kg}$ bw. p. o), followed by induction with Indo $(7.5 \mathrm{mg} / \mathrm{kg}$, s. c) on 8th and 9 th $\mathrm{d}$ of treatment.

Group V: Received SLZ (100 mg/kg b.w., p. o) for $7 \mathrm{~d}$ and followed by induction with Indo $(7.5 \mathrm{mg} / \mathrm{kg}, \mathrm{s}$. c) on 8 th and 9 th $\mathrm{d}$ of treatment.

Drug treatment will be continued till 11th $\mathrm{d}$. On the 12th $\mathrm{d}$ after treatment, animals were anaesthetized with ether and blood was collected by retro-orbital puncture. Later, the animals were sacrificed by cervical dislocation and dissected open to remove the ileum portion. Ileum was flushed gently with saline and cut open. Inflammation was assessed based on the macroscopic scores [7] and quantification of inflammation was done by the assay of serum Lactate Dehydrogenase [8] (LDH) and tissue Myeloperoxidase activity [9] (MPO), GSH [10] and LPO [11].

\section{RESULTS AND DISCUSSION}

Table 1: Effect of Shilajit pre-treatment on macroscopic score, LDH and MPO in Indo induced enterocolitis in rats

\begin{tabular}{lll}
\hline Treatment & Macroscopy (Scoring of ulcers) & LDH (U/l) \\
\hline Normal Control & $1.360 \pm 0.0678$ & $1869 \pm 43.95$ \\
Indomethacin Alone (7.5 mg/kg, s. c) & $7.225 \pm 0.2153^{\# \# \#}$ & $2444 \pm 41.17^{\# \# \#}$ \\
Shilajit Alone (50 mg/kg, p. o) & $1.900 \pm 0.0948$ & $1864 \pm 44.87$ \\
Indo+Shilajit (25 mg/kg, p. o) & $6.060 \pm 0.4020^{*}$ & $2175 \pm 71.89^{*}$ \\
Indo+Shilajit (50 mg/kg, p. o) & $3.975 \pm 0.1019^{* * *}$ & $1806 \pm 12.73^{* * *}$ \\
Indo+SLZ (100 mg/kg, p. o) & $3.325 \pm 0.1392^{* * *}$ & $1750 \pm 0.1640$ \\
\hline
\end{tabular}

Values are expressed as mean \pm SEM, $n=6$. Values of Macroscopic Score are expressed as Points, while LDH is expressed as units/liter and MPO activity as $\mathrm{U} / \mathrm{g}$. \#\#\# $\mathrm{p}<0.001$ significantly different from normal control group. ${ }^{*} \mathrm{p}<0.05,{ }^{* *} \mathrm{p}<0.01$ and ${ }^{* * *} \mathrm{p}<0.001$ significantly different from Indo group. One-way ANOVA followed by Tukey's post test

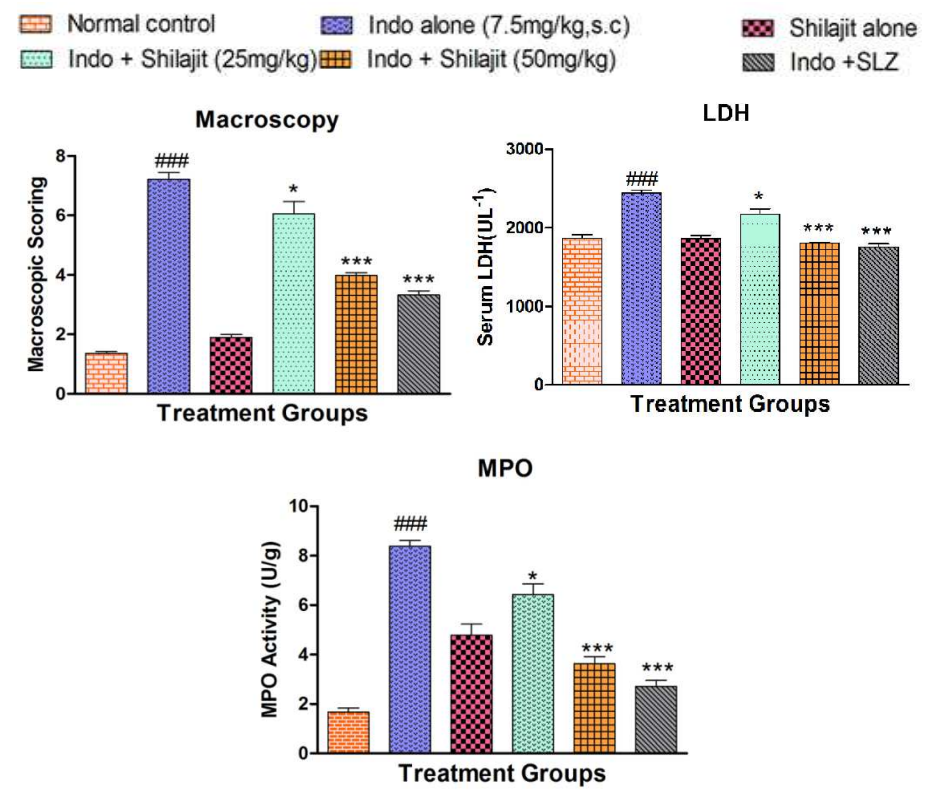

Fig. 1: Effect of Shilajit and SLZ on Macroscopic score, LDH and MPO results in rats treated with Indo. Each bar represents mean \pm SEM, $n=6$. \#\#\# p $<0.001$ significantly different from normal group. ${ }^{*} \mathbf{p}<0.05 * * \mathbf{p}<0.01$ and ${ }^{* * *} \mathbf{p}<0.001$ significantly different from Indo treated group

Table 2: Effect of Shilajit and SLZ on small intestine levels of GSH and LPO content in rats treated with Indomethacin

\begin{tabular}{lll}
\hline Treatment & Glutathione $(\mu \mathrm{mol} / \mathrm{mg}$ of protein) & Tissue LPO (nmol/g of protein) \\
\hline Normal Control & $403.0 \pm 30.57$ & $0.2793 \pm 0.0169$ \\
Indo Alone (7.5 mg/kg, s. c) & $141.5 \pm 6.118^{\# \# \#}$ & $0.6830 \pm 0.0419^{\# \# \#}$ \\
Shilajit Alone $(50 \mathrm{mg} / \mathrm{kg}, \mathrm{p} . \mathrm{o})$ & $386.6 \pm 18.85$ & $0.3103 \pm 0.0271$ \\
Indo+Shilajit $(25 \mathrm{mg} / \mathrm{kg}, \mathrm{p}$. o) & $304.3 \pm 11.21^{*}$ & $0.5130 \pm 0.0459^{*}$ \\
Indo+Shilajit (50 mg/kg, p. o) & $415.7 \pm 22.94^{* * *}$ & $0.4253 \pm 0.0348^{* * *}$ \\
Indo+SLZ (100 mg/kg, p. o) & $490.2 \pm 61.45^{* * *}$ & $0.4025 \pm 0.0110^{* * *}$ \\
\hline
\end{tabular}

Values are expressed as mean \pm SEM, $\mathrm{n}=6$. \#\#\# $\mathrm{p}<0.001$ significantly different from $\mathrm{NC}$ group. ${ }^{*} \mathrm{p}<0.05,{ }^{* *} \mathrm{p}<0.01$ and ${ }^{* * *} \mathrm{p}<0.001$ significantly different from Indo group. One-way ANOVA followed by Tukeys post test 


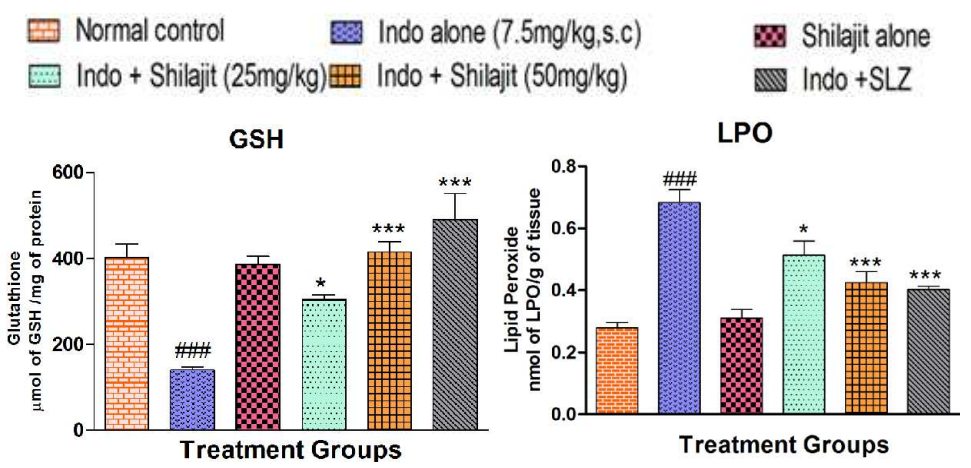

Fig. 2: Effect of Shilajit and SLZ on GSH and LPO results in rats treated with Indo. Each bar represents mean \pm SEM, $n=6$. \#\#\# $p<0.001$ significantly different from normal group. ${ }^{*} p<0.05,{ }^{* *} p<0.01$ and ${ }^{* * *} p<0.001$ significantly different from Indo treated group

\section{RESULTS AND DISCUSSION}

The shilajit and SLZ treated groups showed lower scores compared to Indo alone treated group. An elevation of LDH in serum indicates a shift towards anaerobiosis resulting in the enhanced production of lactic acid. In the present study, serum LDH levels were significantly elevated due to Indo administration compared to normal animals. Pre-treatment with shilajit and SLZ inhibited the elevation of serum LDH level.

MPO is a biochemical marker of neutrophil infiltration in the damaged tissue. In Indo alone treated animals, the MPO activity was significantly elevated. This increase in MPO activity was substantially attenuated in rats pre-treated with shilajit and SLZ. (table 1 and fig. 1)

Antioxidants constitute the foremost defence system that limits the toxicity associated with free radicals. The equilibrium between antioxidants and free radicals is an important process for the effective removal of oxidative stress in intracellular organelles. However, in pathological conditions like enterocolitis, the generation of ROS, lipid peroxidation can dramatically upset this balance with an increased demand on the antioxidant defence system. Present study shows Indo causes increased levels in lipid peroxidation while GSH levels decreased. Pre-treatment of rats with shilajit significantly afforded protection against Indo induced increase of intestinal MDA contents. While the antioxidant power of cell such as GSH content was significantly preserved. (table 2 and fig. 2 ) The protective effect of shilajit is associated with its antioxidant properties, as it acts as ROS scavenger and an inhibitor of lipid peroxidation.

\section{CONCLUSION}

In conclusion, the present study demonstrates that shilajit possess protective effects against Indomethacin-induced enterocolitis and the results may be comparable to that of Sulfasalazine. This protective effect may, at least in part, due to its anti-inflammatory and/or antioxidant actions.

\section{CONFLICT OF INTERESTS}

Declare none

\section{REFERENCES}

1. Baumgart DC, Sandborn WJ. Inflammatory bowel disease: clinical aspects and established and evolving therapies. Lancet 2007;369:1641-57.

2. Scaldaferri F, Fiocchi C. Inflammatory bowel disease: progress and current concepts of etiopathogenesis. J Dig Dis 2007;8:171-8.

3. Head KA, Jurenka JS. Inflammatory bowel disease part i: ulcerative colitis-pathophysiology and conventional and alternative treatment options. Altern Med Rev 2003;8:247-83.

4. Hanan HH, Azza EM. Ameliorative effect of pyrrolidine dithiocarbamate on acetic acid induced colitis in rat. Eur J Pharmacol 2007;554:69-77.

5. Ghosal S, Lal J, Singh SK, Goel RK, Jaiswal AK, Bhattacharya SK. The need for formulation of shilajit by its isolated active constituents. Phytother Res 1991b;5:211-6.

6. Roth MP, Petersen GM, McElree C. Geographic origins of Jewish patients with inflammatory bowel disease. Gastroenterology 1989;97:900-4.

7. Yamada T, Deitch E, Specian RD, Perry MA, Sartor B, MB Grisham. Mechanisms of acute and chronic intestinal inflammation induced by indomethacin. Inflammation 1993;17:641-62.

8. Elson CO, Sartor RB, Tennyson GS, Riddell RH. Experimental models of inflammatory bowel disease. Gastroenterology 1995;109:1344-67.

9. Jagtap AG, Shirke SS, Phadke AS. Effect of polyherbal formulation on experimental models of inflammatory bowel diseases. J Ethnopharmacol 2004;90:195-204.

10. Ellman GI. Tissue sulfahydral groups. Arch Biochem Biophys 1957;80:70-7.

11. Gwlvan G, Saltman P. Different cellular targets of $\mathrm{Cu}$ and $\mathrm{Fe}$ Catalysed Oxidation observed using a $\mathrm{Cu}$ compatible thiobarbiturate acid assay. Biochemica Biophysica Acta 1990;1035:356-60.

\section{How to cite this article}

- $\quad$ Neelima S, Naresh Babu T, Pradeep Kumar M. Effect of shilajit on experimental models of inflammatory bowel disease in rats. Int J Curr Pharm Res 2017;9(4):35-37. 